\title{
Cross-reactive autoantibodies in RA
}

Autoantibodies that recognize posttranslationally modified proteins (known as anti-modified protein antibodies (AMPAs)) are hallmark features of rheumatoid arthritis (RA). New findings published in Annals of the Rheumatic Diseases suggest that these antibodies have a broad specificity and cross-react with several post-translational modifications (PTMs). "These results are highly relevant to further understand and define the antigens that could be recognized in inflamed joints or at other locations in the body that could be involved in the induction and evolution of autoimmunity," says co-first author Theresa Kissel.

AMPAs are typically separated into autoantibody classes on the basis of the PTM they recognize, including citrullination (anti-citrullinated protein antibodies (ACPAs)), carbamylation (anti-carbamylated protein antibodies (ACarPAs)) and acetylation (anti-acetylated protein antibodies (AAPAs)).

In the new study, the researchers generated 14 monoclonal autoantibodies using B cell receptor sequences of autoreactive B cells isolated from patients with RA using citrullinated or acetylated antigens. Notably, all the antibodies recognized at least two different types of PTM, and several of the antibodies recognized all three PTMs. "Most importantly, we show that citrullinated protein-reactive B cells are not only activated upon stimulation with citrullinated antigens, but also after contacting peptides and/or proteins harbouring other modifications, such as acetylation or carbamylation," explains Kissel.

On the basis of these findings, the researchers suggest that ACPAs, ACarPAs and AAPAs should not be categorized into different classes, but should be regarded as one dynamic group of AMPAs. Moreover,

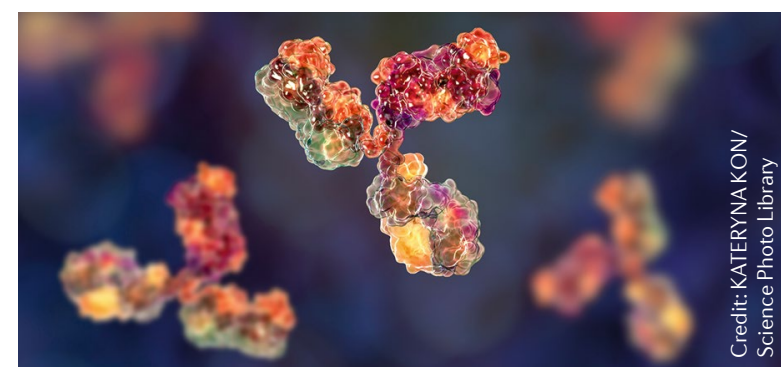

they believe that the use of immunoassays that measure all three PTMs could improve the diagnosis and treatment of RA.

"For future research, it would be interesting to investigate the extent of cross-reactivity in different disease stages, ranging from health to arthralgia, undifferentiated arthritis and RA," remarks co-first author Sanne Reijm. "It would also be relevant to identify the PTM predom-

\section{all the}

antibodies

recognized

at least two

different types of PTM

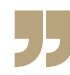
inantly inducing AMPA responses and/or driving inflammation in the synovial compartment in RA."

Jessica McHugh

ORIGINAL ARTICLE Kissel, T. et al. Antibodies and $B$ cells recognising citrullinated proteins display a broad cross-reactivity towards other posttranslational modifications. Ann. Rheum. Dis. https:// doi.org/10.1136/annrheumdis-2019-216499 (2020)

\section{Targeting IL-1 $\beta$ expression in IVDD}

Intervertebral disc degeneration (IVDD), a leading cause of low back pain, is primarily mediated by inflammatory responses induced by pro-inflammatory cytokines such IL-1 $\beta$. New findings suggest that IL- $1 \beta$ can promote its own expression in IVDD via a positive feedback loop involving the NLRP3 inflammasome, which can be targeted therapeutically with melatonin.

The NLRP3 inflammasome is a known source of IL-1 $\beta$ and has been implicated in several inflammatory diseases. In the new study, IL-1 $\beta$ upregulated the expression of NLRP3 and p20 (components of the NLRP3 inflammasome) in vitro in nucleus pulposus (NP) cells (cells that make up part of the intervertebral disc). Furthermore, NLRP3 inflammasome activation (including the expression of NLRP3, p20 and IL-1 $\beta$ ) was upregulated in the degenerative discs of patients with IVDD and in rats with annulus fibrosus puncture-induced IVDD (a rat model of IVDD).
Further experiments found that IL-1 $\beta$ could induce both NLRP3 inflammasome priming and activation in NP cells by upregulating nuclear factor $-\kappa B$ $(N F-\kappa B)$ signalling. Indeed, treatment with an inhibitor of the NF- $\kappa B$ pathway (SM7368) inhibited IL-1 $\beta$-induced upregulation of NLRP3 expression. IL- $1 \beta$ could also promote the production of mitochondrial reactive oxygen species (mtROS) in these cells in vitro.

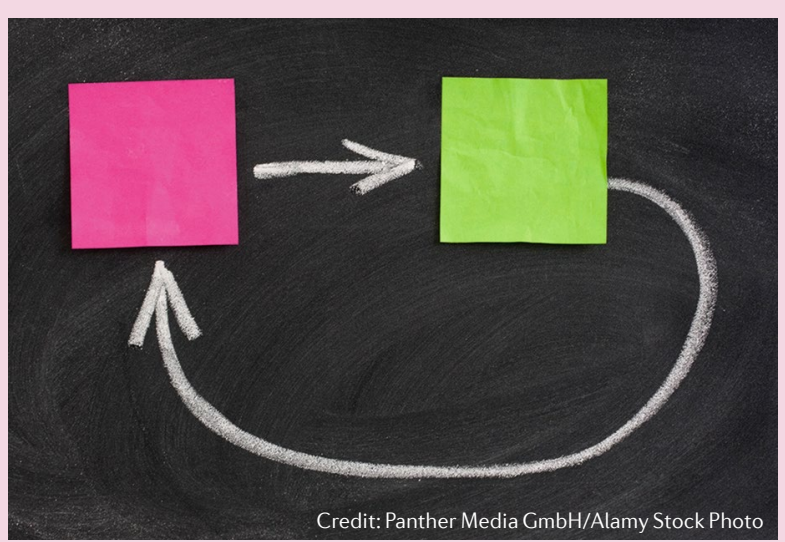

Melatonin is a neuroendocrine hormone with various functions, including anti-inflammatory activities. In vitro treatment of NP cells with melatonin inhibited the positive feedback loop of IL-1 $\beta$, including IL- $1 \beta$-induced NF- $\kappa B$ signalling, NLRP3 inflammasome priming and activation, and $\mathrm{mtROS}$ production.

Notably, in the rat model of IVDD, treatment with melatonin also disrupted this positive feedback loop, alleviating the progression of IVDD and IVDD-related low back pain.

"We plan to knock down NLRP3 to further verify the mechanisms of IL-1 $\beta$ metabolism," explains Jianru Wang, co-corresponding author of the study. "We think that melatonin could be a promising therapeutic strategy for patients with IVDD and low back pain," continues co-corresponding author Zhaomin Zheng.

Jessica McHugh

ORIGINAL ARTICLE Chen, F. et al. Melatonin alleviates intervertebral disc degeneration by disrupting the IL-1 $\beta / N F-\kappa B-N L R P 3$ inflammasome positive feedback loop. Bone Res. 8, 10 (2020) 